\title{
Robotic Walker for Slope Mobility Assistance with Active-Passive Hybrid Actuator
}

\author{
Junqiang Li ${ }^{*} \mathbb{0}$, Lei Zhao and Tiejun Li
}

\begin{abstract}
Walking assistance can be realized by active and passive robotic walkers when their users walk on even roads. However, fast signal processing and real-time control are necessary for active robotic walkers when the users walk on slopes, while assistive forces cannot be provided by passive robotic walkers when the users walk uphill. A robotic walker with an active-passive hybrid actuator (APHA) was developed in this study. The APHA, which consists of a rotary magnetorheological (MR) brake and a DC motor, can provide mobility assistance to users walking both uphill and downhill via the cooperative operation of the MR brake and DC motor. The rotary MR brake was designed with a T-shaped configuration, and the system was optimized to minimize the brake volume. Prototypes of the APHA and robotic walker were constructed. A control algorithm for the robotic walker was developed based on the characteristics of the APHA and the structure of the robotic walker. The mechanical properties of the APHA were characterized, and experiments were conducted to evaluate the mobility assistance supplied by the robotic walker on different roads. The results show that the APHA can meet the requirements of the robotic walker, and suitable assistive forces can be provided by the robotic walker, which has a simple mechanical structure and control method.
\end{abstract}

Keywords: Robotic walker, Active-passive hybrid actuator, MR brake, Slope mobility assistance

\section{Introduction}

The rapid growth of the elderly population in today's society has resulted in an increase in the proportion of elderly people who require assistance in their daily living. Safe and independent walking is an important part of improving their quality of life $[1,2]$. Freedom of movement should be available to individuals so that they can lead their lives as normally as possible. The ability to walk independently helps to sustain social interactions and is therefore beneficial for both physical and mental health. It is therefore important to provide tools that preserve the walking ability of the elderly. To encourage independent walking, researchers have developed various walking assistance devices such as canes [3, 4], walkers [5], and exoskeleton robots [6,7]. A walker is a widely used device for elderly users who need additional support to maintain

\footnotetext{
*Correspondence: lijunqiang@hebut.edu.cn

School of Mechanical Engineering, Hebei University of Technology, Tianjin 300401, China
}

balance and stability while walking. Various types of robotic walkers have been developed to overcome the shortcomings of conventional walkers.

Robotic walkers can be divided into the two main categories of active and passive walkers according to their actuators. Active robotic walkers use servo motors to guide the user $[8,9]$. They therefore need to interpret the user's walking intentions through human-machine interfaces. The human-machine interface requires one or more types of sensors such as force sensors [10,11], cameras [12], or laser sensors [13, 14]. During the walking process, the servo motor should be controlled in real time [15]. Passive robotic walkers move via forces supplied by the user and use controlled brakes, such as electromechanical $[16,17]$ or magnetorheological (MR) brakes $[18,19]$, to steer or stop the walker in case of emergencies. Movements on level roads can be achieved using both active and passive robotic walkers. Uphill and downhill roads are commonly encountered in outdoor environments in daily life, but they can be difficult and 
dangerous for the elderly to traverse. Forces can be provided by active robotic walkers to assist with walking on uphill and downhill roads; however, to effectively interpret the user's walking intentions, fast signal processing and real-time control are crucial for practical robotic walkers [20]. Walking downhill with a passive robotic walker is generally safe because of energy dissipation, but assistive forces cannot be provided by passive robotic walkers when walking uphill.

In this study, a robotic walker with an active-passive hybrid actuator (APHA) is developed. The APHA is composed primarily of a rotary MR brake and a DC motor. By coordinating the MR brake and DC motor, both passive and active torques can be generated by the APHA. through the APHA and the control algorithm, the robotic walker can provide assistance for both uphill and downhill walking.

\section{Principle of Slope Mobility Assistance}

The APHA is designed to supply force for slope mobility assistance. The APHA has several components, which include a rotary MR brake, a worm gear reducer, a slip ring, and a DC motor, as shown in Figure 1.

The worm gear reducer is placed between the DC motor and the MR brake, and a flange is used to connect the worm gear reducer with the MR brake shell. When the DC motor is turned off, the self-locking function of the worm gear reducer constrains the MR brake shell so that passive torque can be obtained on the MR brake shaft. When the DC motor is driven to rotate the MR brake shell and the shaft speed is less than the shell speed, active torque can be generated. The values of the passive and active torques are determined by the MR brake excitation current. In addition, the direction of the active torque depends on that of the DC motor rotation.

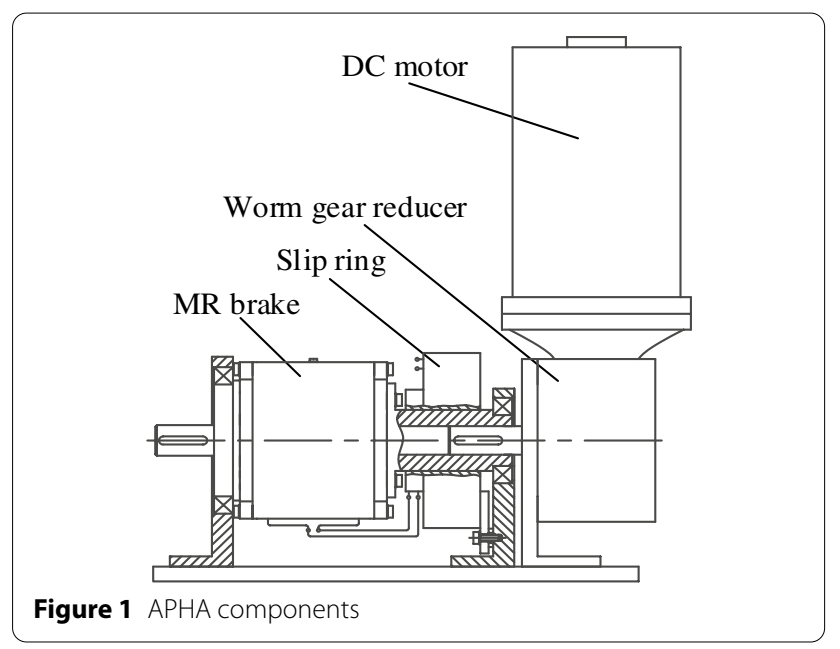

A slip ring is installed between the MR brake and the worm gear reducer. The slip-ring rotor is connected to the flange, and the stator is fixed. The wires of the MR brake are connected to the connector of the slip ring rotor to realize continuous rotation of the MR brake shell. The rotary MR brake is discussed in the next section.

A robotic walker outfitted with the APHA is shown in Figure 2. The purpose of this study is to provide an assistive force for the user of the robotic walker so that the loads exerted on the user while walking uphill and downhill are similar to the loads the user experiences while walking on a level surface.

The front wheel of the robotic walker is driven by the APHA, and two casters are installed on the back of the robot. When walking uphill, the MR brake shell is driven by the DC motor through the worm gear reducer at a shell speed that is designed to be greater than the shaft speed. The yield stress of the MR fluid in the MR brake can be controlled by the excitation current. An active assistive force can then be produced on the MR brake shaft, and the direction of the force is determined by the rotation direction of the DC motor. When walking downhill, the DC motor is stopped, and the MR brake shell is constrained by the worm gear reducer. An assistive force can be obtained by driving the MR brake with an excitation current. When walking on a level surface, no energy needs to be provided by the system, in which case the robotic walker works just like a conventional walker. The driving wheel at the front of the walker and two casters in the back allow the user to

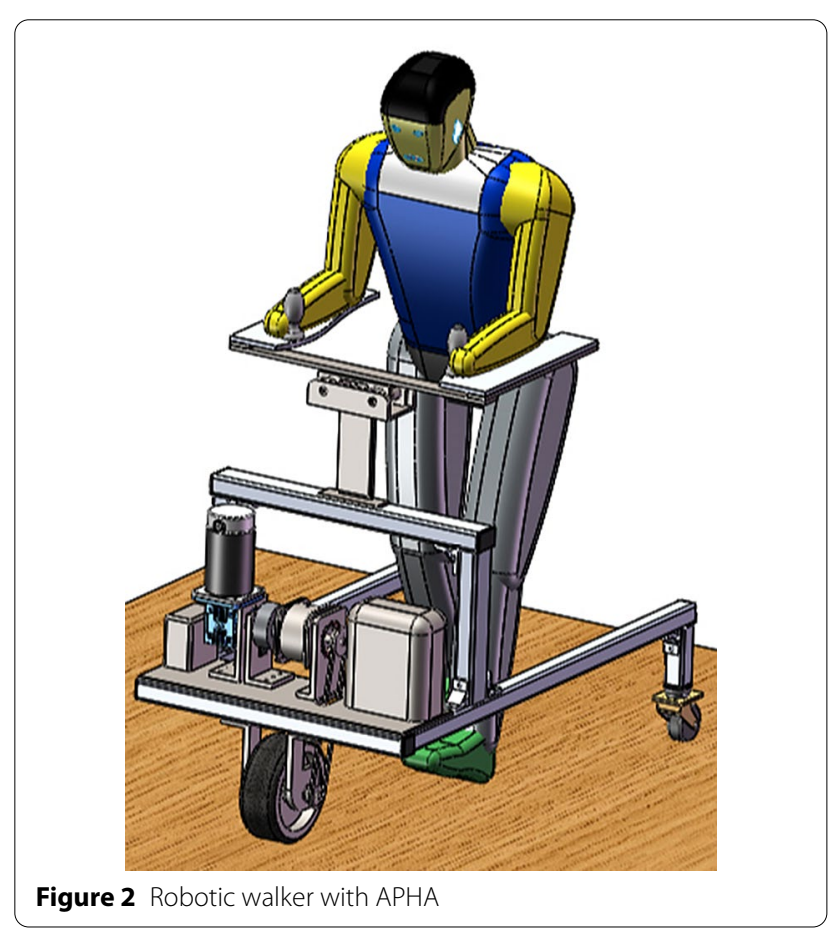


change the direction of the robotic walker with his own force.

Based on the configuration of the robotic walker, the speed of the robotic walker $\left(V_{w}\right)$ can be calculated as

$$
V_{w}=\frac{2 \pi R_{w} \cdot n_{s}}{60 \cdot i}
$$

where $R_{w}$ is the front wheel radius, $n_{s}$ is the number of revolutions per minute of the MR brake shaft, and $i$ is the reduction ratio.

On both uphill and downhill roads, the force on the human user and the robotic walker due to gravity can be compensated by an equal force. Considering the rolling resistance shown in Figure 3, the required assistive forces can be obtained as

$$
\left\{\begin{array}{l}
F=-\left(m_{h}+m_{r}\right) \cdot g \cdot \sin (\theta)-F_{r}, \\
F^{\prime}=-\left(m_{h}+m_{r}\right) \cdot g \cdot \sin (\theta)+F_{r},
\end{array}\right.
$$

where $F$ is the assistive force supplied by the robotic walker on an uphill road, $F^{\prime}$ is the assistive force on a downhill road, $m_{h}$ is the mass of the user, $m_{r}$ is the mass of the robotic walker, $g$ is the acceleration due to gravity, $\theta$ is the angle of the inclined plane (the value of $\theta$ can be measured using a tilt sensor in real time), and $F_{r}$ is the rolling resistance (the rolling resistance is usually assumed to be $1 / 100$ of the normal force) [21]. Thus, the rolling resistance of the robotic walker can be calculated as

$$
F_{r}=\frac{m_{r} \cdot g \cdot \cos (\theta)}{100} .
$$

On uphill roads, the torque that should be supplied by the APHA, $T_{a}$, can be obtained as

$$
T_{a}=\frac{|F| \cdot R_{w}}{i} .
$$

On downhill roads, the torque that should be supplied by the APHA, $T_{a}^{\prime}$, can be obtained as

$$
T_{a}^{\prime}=\frac{\left|F^{\prime}\right| \cdot R_{w}}{i}
$$

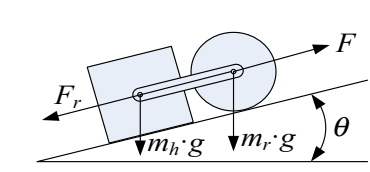

(a) Uphill road

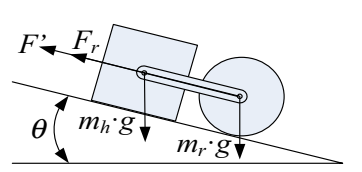

(b) Downhill road
Figure 3 Forces on different roads
It can be determined from Eqs. (4) and (5) that

$$
T_{a}>T_{a}^{\prime}
$$

The robotic walker should supply sufficient assistive force for the user on both uphill and downhill roads. Therefore, the torque that should be supplied by the APHA is given by Eq. (4).

The slopes in different public facility slopes have different gradients. According to the design code for civil buildings in China(GB50352-2005), the largest gradient for wheelchair slopes should not exceed $1 / 8$, that is, the inclination angle of the slope should be less than $7.13^{\circ}$. Thus, the slope inclination angle used in this study is $\theta=8^{\circ}$.

To supply sufficient assistive force for most persons, $m_{h}$ was set to $85 \mathrm{~kg}$, the mass of the robotic walker was estimated to be $m_{r}=20 \mathrm{~kg}$, and the front wheel radius and the reduction ratio were set to $R_{w}=100 \mathrm{~mm}$ and $i=5 / 9$ in this study. The corresponding value of $T_{a}$ was calculated to be $T_{a}=26.1 \mathrm{~N} \cdot \mathrm{m}$. The actual value of the MR brake torque is usually smaller than the theoretical result, so $T_{a}$ was set to $30.0 \mathrm{~N} \cdot \mathrm{m}$.

\section{MR Brake for APHA}

\subsection{MR Brake Structure}

A MR brake is a type of controlled brake that creates a torque by changing the viscosity of the MR fluid inside the brake. The apparent viscosity of the MR fluid can be changed very quickly (in milliseconds) by applying a magnetic field [22]. The braking torque of the rotary MR brake is generated by the shear stress of the MR fluid placed in the gap between the two rotating surfaces [23]. The MR fluid can be modeled using nonlinear models such as the Bingham plastic model [24]:

$$
\left\{\begin{array}{cl}
\tau=\tau_{y}(H) \operatorname{sgn}(\dot{\gamma})+\mu \dot{\gamma}, & \tau>\tau_{y}, \\
\dot{\gamma}=0, & \tau \leq \tau_{y},
\end{array}\right.
$$

where $\tau$ is the shear stress, $H$ is the magnetic flux intensity, $\dot{\gamma}$ is the shear rate, $\tau_{y}$ is the yield stress, which changes with the magnetic flux intensity, and $\mu$ is the plastic viscosity of the MR fluid.

Different types of MR fluids exhibit particular characteristics according to their rheological properties. The MR fluid used in this study is MRF-140CG, which was acquired from the Lord Corporation.

The MR brake structure determines the direction of the magnetic field lines. To achieve torques with smaller magnetomotive forces in the brake, the MR brake should have a large effective area and a short magnetic circuit in the rotor and shell. There are several types of MR brakes with different rotor shapes such as the disc-type [25], multiple disc-type [26], and drum-type [27] MR brakes. 


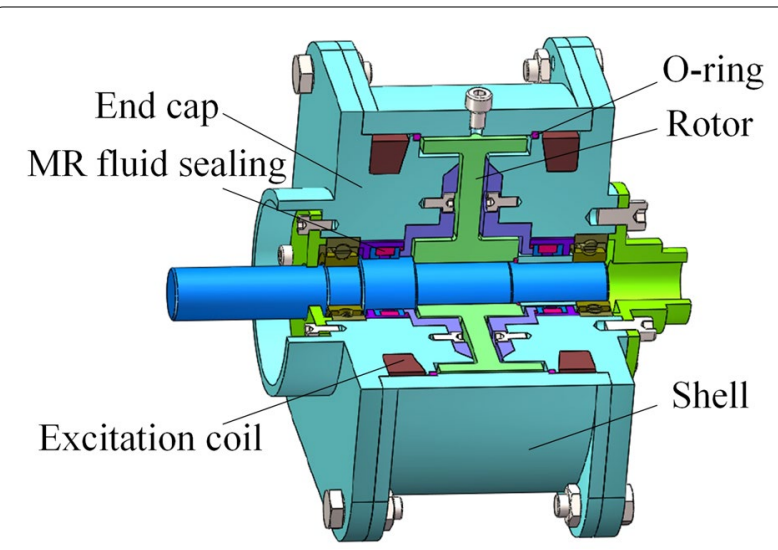

Figure 4 MR brake structure

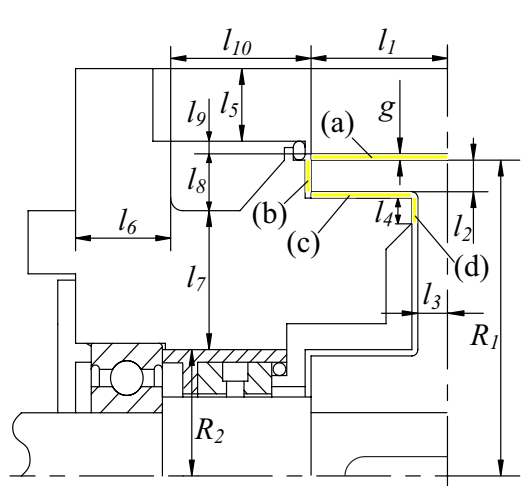

Figure 5 Structural parameters

To achieve a larger torque in a rotary MR brake without increasing the size of the brake, a larger surface in the MR brake rotor should be activated by the magnetic flux. This objective is achieved in this study by designing the rotor with a $\mathrm{T}$-shape and strategically placing excitation coils to provide a larger effective area on the surface of the rotor. Figure 4 shows a diagram of the MR brake components. The designed rotary MR brake consists of four main part, namely, the MR fluid, the MR fluid sealing, the rotor, and the stator. The magnetic field lines pass through the stator, MR fluid, and rotor and form a closed loop. The rotor of the brake is T-shaped so that the brake has multiple effective areas as shown in Figure 5, where areas (a) to (d) are respectively called effective areas 1 to 4. The rotor serves as the steel core for the electromagnet and the transmission element for the torque. Excitation coils are placed in the end caps that are installed on both ends of the shell, and the end caps and shell are connected to form the stator.

The two excitation coils generate magnetic fields in opposite directions to form a magnetic circuit with two parallel magnetic paths. The magnetic path is kept short because a short magnetic path can effectively reduce the volume and weight of the brake.

A sealing approach using MR fluid sealing is applied on both sides of the brake shaft. The MR fluid itself functions as the sealing element. Compared to a conventional dynamic seal, MR fluid sealing has a long lifetime and a good sealing effect. Two O-rings are employed between the end caps and the shell, and the gap between the stator and rotor is filled by MR fluid.

\subsection{Torque Calculation and Design Optimization of MR Brake}

DT4E-type magnetic pure iron was chosen as the magnetic core material to obtain reasonable values of the MR brake structural parameters. The yield stress of the MR fluid and the magnetic induction intensity of the magnetic core material should be determined based on the maximum brake torque. The required magnetic field intensity and the magnetic induction intensity of the MR fluid can then be obtained from the manufacturer technical data sheet, while the magnetic field strength of the magnetic core material can be obtained from a material handbook [28]. The brake parameters for each part are shown in Figure 5.

The magnetic flux around the magnetic circuit is constant and has the value of $\Phi=B \cdot S$. Based on Ohm's law for magnetic circuits, two principles should be followed in the magnetic circuit calculations: (1) parts that have the same magnetic conductivity should have equal magnetic flux areas, and (2) the ratio of the magnetic flux areas of parts that have different magnetic conductivities should be equal to the reciprocal of the ratio of their magnetic induction intensities in the initial design. According to these two magnetic circuit calculation principles, the relationships between the structural parameters of the MR brake can be expressed as

$$
\left\{\begin{array}{l}
\frac{S_{1}}{S_{5}}=\frac{B_{t}}{\sigma B_{y}}, \\
S_{1}=S_{2}+S_{3}+S_{4}, \\
S_{5}=S_{6},
\end{array}\right.
$$

where $B_{t}$ is the magnetic induction intensity of the magnetic pure iron, $B_{y}$ is the magnetic induction intensity of the MR fluid in the effective area, and

$$
\begin{aligned}
& S_{1}=2 \pi R_{1} l_{1}, \\
& S_{2}=\pi R_{1}^{2}-\pi\left(R_{1}-l_{2}\right)^{2}, \\
& S_{3}=2 \pi\left(R_{1}-l_{2}-\mathrm{g}\right)\left(l_{1}-l_{3}-g\right),
\end{aligned}
$$




$$
\begin{aligned}
& S_{4}=\pi\left(R_{1}-l_{2}-g\right)^{2}-\pi\left(R_{1}-l_{2}-g-l_{4}\right)^{2}, \\
& S_{5}=\pi\left(R_{1}+l_{9}+g+l_{5}\right)^{2}-\pi\left(R_{1}+g+l_{9}\right)^{2}, \\
& S_{6}=2 \pi\left(R_{2}+l_{7}\right) l_{6},
\end{aligned}
$$

where $\sigma$ is the magnetic flux leakage coefficient. The inequality

$$
S_{7} \geq S_{6}
$$

where $S_{7}=\pi\left(R_{2}+l_{7}\right)^{2}-\pi R_{2}^{2}$ should also be simultaneously satisfied.

The design configuration of the MR brake is optimized to maximize the braking torque and minimize the weight. Because the operational mode of the MR fluid in the brake corresponds a shear model, the braking torque of the MR brake can be calculated using the Bingham plastic model of a MR fluid and the configuration parameters of the MR brake. The plastic viscosity of the MR fluid is ignored because the speed of revolution of the MR brake is low. The braking torques occur in effective areas $1,2,3$, and 4 , and can be calculated using the following formulae:

$$
\left\{\begin{array}{l}
T_{1}=\tau_{1} S_{1}\left(R_{1}+0.5 g\right), \\
T_{2}=\int_{r_{1}}^{r_{2}} \tau_{2} \cdot 2 \pi r^{2} \cdot \mathrm{d} r \\
T_{3}=\tau_{3} S_{3}\left(R_{1}-l_{2}-0.5 g\right), \\
T_{4}=\int_{r_{3}}^{r_{4}} \tau_{4} \cdot 2 \pi r^{2} \cdot \mathrm{d} r
\end{array}\right.
$$

where $\tau_{1}, \tau_{2}, \tau_{3}$, and $\tau_{4}$ are the yield stresses of the MR fluid, and

$$
\begin{aligned}
& r_{1}=R_{1}-l_{2}, \\
& r_{2}=R_{1}, \\
& r_{3}=R_{1}-l_{2}-g-l_{4}, \\
& r_{4}=R_{1}-l_{2}-g .
\end{aligned}
$$

The braking torque of the brake can be obtained by combining the torques from the four effective areas:

$$
T=2 \cdot\left(T_{1}+T_{2}+T_{3}+T_{4}\right) .
$$

The volume of the brake can then be calculated using the radius of the rotor $\left(R_{1}\right)$ and Eqs. (8), (10), and (11). A given constraint torque can be satisfied by multiple MR structures with different volumes $(V)$ and different values of $R_{1}$; therefore, there may exist a value of
Table 1 Parameter values

\begin{tabular}{lclc}
\hline Parameter & Value & Parameter & Value \\
\hline$T(\mathrm{~N} \cdot \mathrm{m})$ & 30.0 & $I_{3}(\mathrm{~mm})$ & 3.0 \\
$\tau_{1}(\mathrm{kPa})$ & 45.0 & $I_{4}(\mathrm{~mm})$ & 2.0 \\
$\tau_{2}(\mathrm{kPa})$ & 45.0 & $I_{8}(\mathrm{~mm})$ & 9.0 \\
$\tau_{3}(\mathrm{kPa})$ & 45.0 & $I_{9}(\mathrm{~mm})$ & 2.5 \\
$\tau_{4}(\mathrm{kPa})$ & 45.0 & $I_{10}(\mathrm{~mm})$ & 14.0 \\
$B_{t}(\mathrm{~T})$ & 1.50 & $R_{2}(\mathrm{~mm})$ & 16.0 \\
$B_{y}(\mathrm{~T})$ & 0.75 & $g(\mathrm{~mm})$ & 1.0 \\
$I_{2}(\mathrm{~mm})$ & 4.0 & $\sigma$ & 1.10 \\
\hline
\end{tabular}

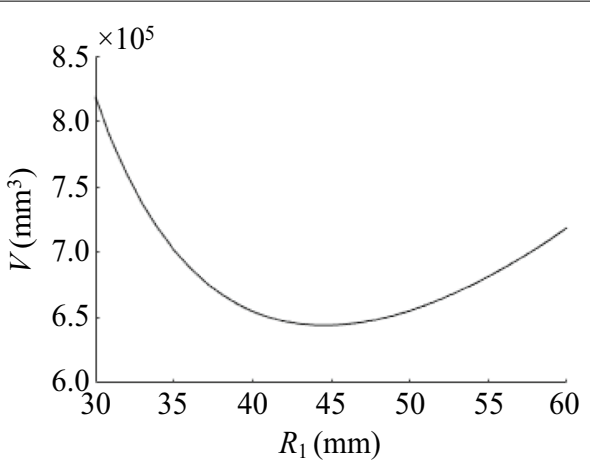

Figure 6 Relationship between $V$ and $R_{1}$

$R_{1}$ at which the volume is minimized. The relationship between $V$ and $R_{1}$ can be represented as

$$
V=\pi\left(R_{1}+g+l_{9}+l_{5}\right)^{2} \cdot\left(l_{1}+l_{6}+l_{10}\right) \cdot 2,
$$

where the parameters are shown in Figure $5 . l_{1}, l_{5}$, and $l_{6}$ can be calculated using Eqs. (8), (10), and (11) after the other parameter values are predefined to the values shown in Table 1.

Under the constraint $R_{1} \in[30,60]$, the value of $V$ was calculated with MATLAB. The relationship between $V$ and $R_{1}$ is shown in Figure 6.

Figure 6 shows that as $R_{1}$ increases, the volume first decreases and then increases. There is therefore a value of $R_{1}$ at which the volume is minimized. This particular value was calculated using MATLAB. It was confirmed that inequality (9) is satisfied at this value of $R_{1}$. The parameters for the MR brake are hence obtained.

Using the structural parameters of the brake, the magnetomotive force $(F)$ can be calculated as

$$
F=I N=\sum_{i=1}^{6} H_{i} L_{i},
$$


where $N$ is the number of coil turns, $I$ is the current in the coil, and $L$ is the length of the magnetic path.

The calculated value of $F$ is $F=240 \mathrm{AN}$. Considering the installation space of the coil in the MR brake, $N$ was set to $60 \mathrm{~N}$, and $I$ to $4.0 \mathrm{~A}$.

\section{Prototype of Robotic Walker}

The robotic walker was designed with a three-wheel structure, as shown in Figure 7. The front wheel is the driving wheel, the rear wheel is the driven wheel, and a force sensor is set between the support plate and the walker body to sense the force between the user and the walker. The total weight of the robot is $22.3 \mathrm{~kg}$.

Figure 8 shows the system configuration of the robotic walker prototype. To adapt the walker to different users, a potentiometer is used to sense the weight of the user whereby the output voltage from the potentiometer corresponds to the weight of the user. An NI myRIO-1900 (from NI, Inc.) is used as the controller for the robotic walker, and a tilt sensor inside the controller is used to obtain the slope angle of the road. The pulse-width modulated (PWM) control signal from the myRIO to the MR brake is amplified using the MR brake driver, which is implemented using an advanced motion control brush servo amplifier. The on-off control signals from the myRIO to the DC motor are translated using a relay and a contactor. The APHA drives the driving wheel through a chain drive to provide an assistive force for human walking.

A control strategy was designed based on the torque characteristics of the APHA. The control flowchart is shown in Figure 9. A potentiometer is used to set the magnitude of the assistive force to suit the user based

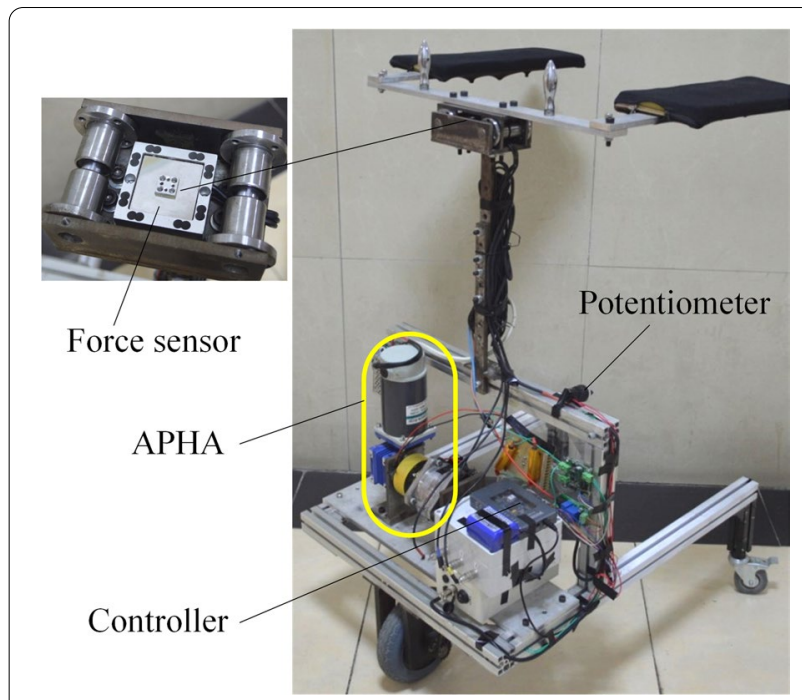

Figure 7 Prototype of robotic walker

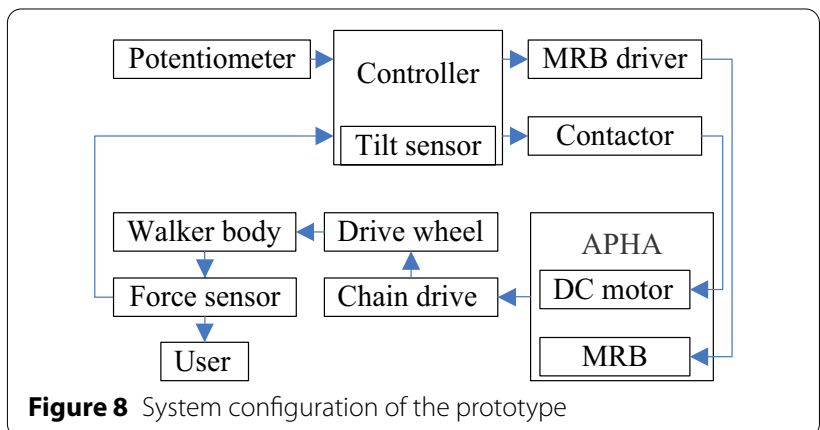

on his weight. The tilt sensor is used to obtain the slope angle of the road surface when the user begins to walk.

In practical applications, the movement state of the user can be classified into the three states of even roads, uphill roads, and downhill roads. The following method is used to obtain the required assistive force.

(1) To prevent the walker from starting and stopping frequently, a road with an absolute inclination angle of less than $1.0^{\circ}$ (threshold $=1.0^{\circ}$ ) is classified as a

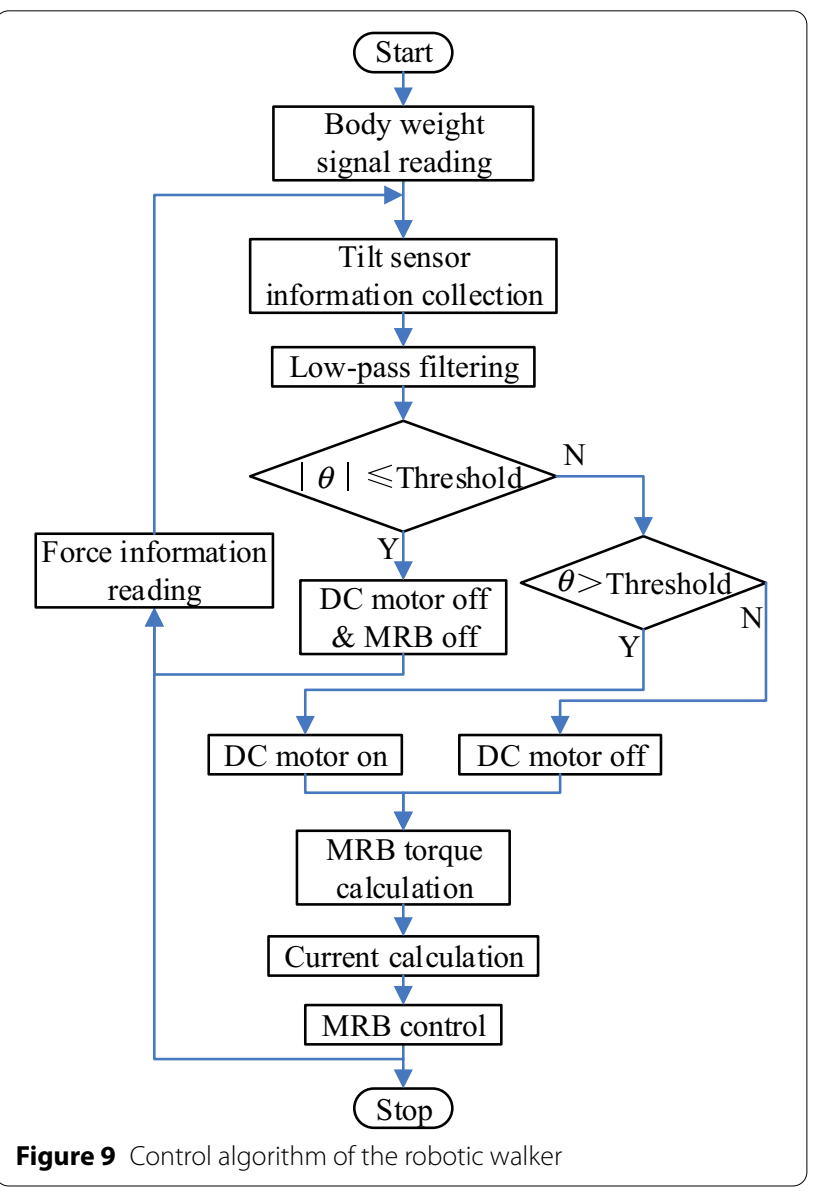


horizontal road. In this case, the controller system does not output the control quantity, and the movement of the walker is driven by the force from the user in a similar manner to traditional walkers.

(2) The structural parameters of the robotic walker are constant whereas the mass of the user can be set. Therefore, the required torque from the APHA can be calculated using the slope angle measured by the tilt sensor. Moreover, because the torque of the APHA supplied by the MR brake and the torque of the MR brake are related to the current, the required current can be calculated. When the user walks on uphill roads, the control system controls the active torque output from the APHA based on the user's weight and the slope angle to generate a force to assist the user. The force is oriented along the walking direction. The control current of the brake in the APHA is given by

$\left\{\begin{array}{l}T_{a}=\frac{\left(100 \cdot\left(m_{h}+m_{r}\right) \cdot g \cdot \sin (\theta)+m_{r} \cdot g \cdot \cos (\theta)\right) \cdot R_{w}}{100 \cdot i}, \\ I=0.0003 \cdot T_{a}^{3}-0.0101 \cdot T_{a}^{2}+0.1899 \cdot T_{a}-0.1387,\end{array}\right.$

where $T_{a}$ is calculated using Eq. (4), and the relation between $T_{a}$ and $I$ was acquired from the APHA torque test results, which are shown in Table 2.

(3) When walking downhill, the control system controls the passive torque output from the APHA to generate a force to assist the user. The force is oriented in the opposite direction to the walking direction. The control current of the brake in the APHA is given by

$$
\left\{\begin{array}{l}
T_{a}^{\prime}=\frac{\left(100 \cdot\left(m_{h}+m_{r}\right) \cdot g \cdot \sin (\theta)-m_{r} \cdot g \cdot \cos (\theta)\right) \cdot R_{w}}{100 \cdot i}, \\
I^{\prime}=0.0003 \cdot\left(T_{a}^{\prime}\right)^{3}-0.01 \cdot\left(T_{a}^{\prime}\right)^{2}+0.1851 \cdot T_{a}^{\prime}-0.0851 .
\end{array}\right.
$$

Table 2 Torque test results

\begin{tabular}{|c|c|c|}
\hline Current (A) & Passive torque $(\mathrm{N} \cdot \mathrm{m})$ & $\begin{array}{l}\text { Active } \\
\text { torque } \\
(\mathrm{N} \cdot \mathrm{m})\end{array}$ \\
\hline 0.0 & 0.6 & 0.5 \\
\hline 0.5 & 4.3 & 3.9 \\
\hline 1.0 & 9.4 & 9.5 \\
\hline 1.5 & 14.4 & 14.2 \\
\hline 2.0 & 18.9 & 18.5 \\
\hline 2.5 & 22.1 & 21.8 \\
\hline 3.0 & 23.9 & 23.4 \\
\hline 3.5 & 25.6 & 25.6 \\
\hline 4.0 & 27.2 & 26.8 \\
\hline
\end{tabular}

The current values ( $I$ and $I^{\prime}$ ) should not be less than $0 \mathrm{~A}$ when the robotic walker is operating. Therefore, the current values $(I$ and $I)$ are set to $0 \mathrm{~A}$ when the calculated current values are less than $0 \mathrm{~A}$. The control program was programmed with LabVIEW, and the control cycle interval was set to $40 \mathrm{~ms}$.

\section{Experiments and Results}

\subsection{MR Brake Structure}

After the optimized design and mechanical performance analysis of the MR brake were completed, the MR brake was manufactured, and the APHA was constructed. The APHA prototype was installed in the experimental setup shown in Figure 10.

A servo motor (from Yaskawa, Inc.) with a gear reducer (7:1) was used to generate the shaft rotation. The gear reducer shaft was connected to a shaft-to-shaft rotary torque sensor (Beijing YuHang Meter Science and Technology Co. Ltd.). The other end of the torque sensor was connected to the APHA prototype. Flexible couplings were used to connect the various components. The servo motor was controlled by an IPC with a control board (Advantech Inc.), and the output signal of the torque sensor was connected to a data acquisition board (Advantech Inc.).

\section{(1) Passive torque tests.}

In this experiment, the DC motor was turned off, and the shaft of the brake was driven by the servo motor. The rotational speed was maintained at $30 \mathrm{r} / \mathrm{min}$, and the corresponding robotic walker speed was $0.57 \mathrm{~m} / \mathrm{s}$. The shell of the brake could not rotate because of the self-locking of the worm gear reducer. The current applied to the coil was manually increased from 0 to $4.0 \mathrm{~A}$ at intervals of 0.5 A. The data for each step were taken over a few intervals to achieve consistency in the readings. The results are presented in Table 2.

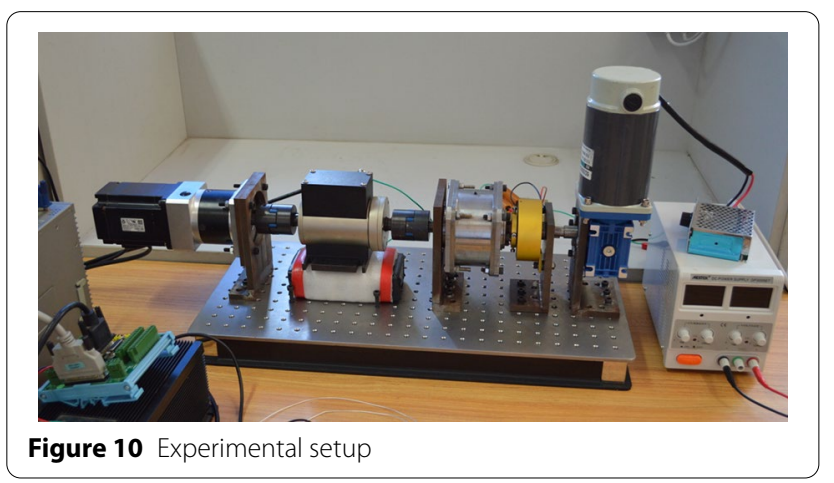




\section{(2) Active torque tests.}

In this experiment, the DC and MR brakes worked simultaneously. The DC motor drove the brake shell at 40 $\mathrm{r} / \mathrm{min}$ with a DC voltage of $24 \mathrm{~V}$ and current of $3 \mathrm{~A}$. The servo motor drove the brake shaft to rotate in the same direction as the shell. The brake shaft was driven by the servo motor at $30 \mathrm{r} / \mathrm{min}$. The current was applied to the coil and increased in intervals of $0.5 \mathrm{~A}$ from 0 to $4.0 \mathrm{~A}$. The results are shown in Table 2.

The relationship between the torque and current is shown in Figure 11. It can be seen from Figure 11 that the results from the passive torque test are very close to those from the active torque test at the same currents. This is because both the passive and active torques were generated by the MR brake while the DC motor rotation direction only determined the active torque direction. When the current was $0 \mathrm{~A}$, the passive torque was $0.6 \mathrm{~N} \cdot \mathrm{m}$. This implies that the user will suffer less resistance as he walks on a level road. When the current was $4.0 \mathrm{~A}$, the passive and active torques were $27.2 \mathrm{~N} \cdot \mathrm{m}$ and $26.8 \mathrm{~N} \cdot \mathrm{m}$, respectively. These results demonstrate that the performance of the APHA meets the requirements of the robotic walker.

\subsection{Slope Mobility Assistance Experiments}

An experimental platform for the robotic walker was constructed to determine the interaction forces between the human and the machine under different road conditions. The results were used to verify the assistive effects of the robotic walker. Figure 12 shows the platform, which has an inclination of $8^{\circ}$ for both the uphill and downhill slopes.

During the experiment, the user first walked on the even road with the robotic walker, followed by the uphill road, the even road, the downhill road, and finally, the

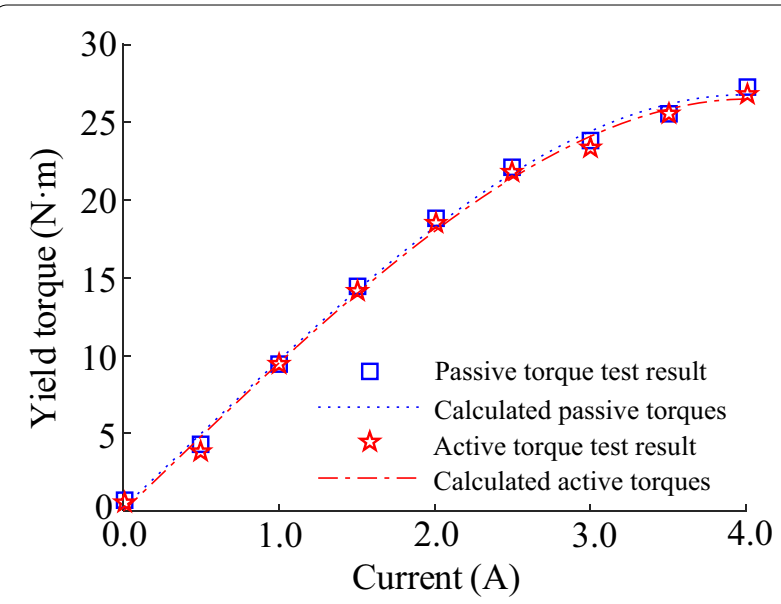

Figure 11 Relation between torque and current

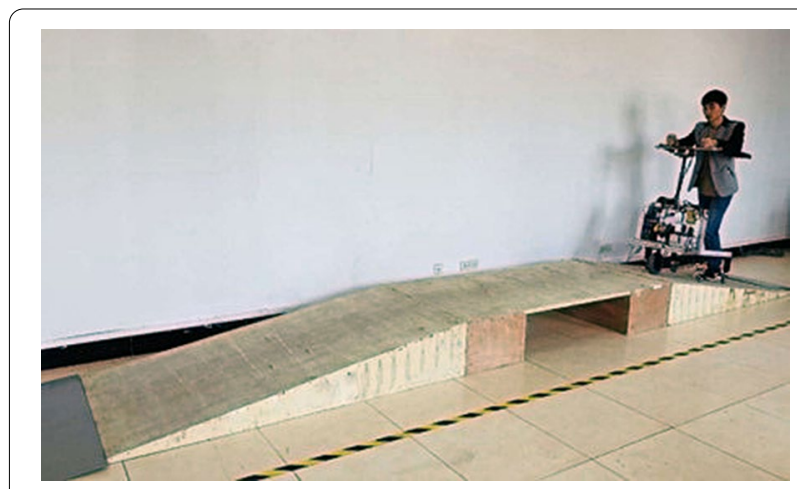

Figure 12 Experimental platform

even road in the stated order. The user's weight was set to $70 \mathrm{~kg}$, and the force sensor was used to sense the humanrobotic walker interaction. The experimental results are shown in Figure 13. In Figure 13, (1) is the desired assistive force calculated by Eqs. (4) and (5), (2) is the experimental assistive force measured by the force sensor, and (3) is the tilt sensor output.

The experimental platform was built with planks. After many experiments, the platform surface became uneven. The experimental results show that in the uphill and downhill sections, the robotic walker can accurately sense the road slope, and the control system can control the APHA according to the road angle so that the walker can provide an assistive force for the user.

The measured assistive forces were lower than but similar to the desired assistant forces. This discrepancy might be due to numerous causes such as the frictional forces in the transmission mechanism of the robotic walker and the force sensing mechanism. It should be noted that setting a larger body weight value results in a larger assistive force. Because of the frictional force and the passive torque of the APHA at $0 \mathrm{~A}$, the force required to move the robotic walker when walking on a level road is approximately $16 \mathrm{~N}$.

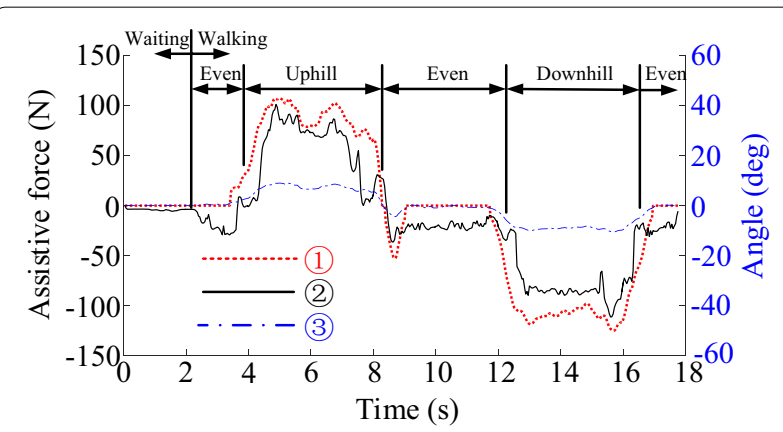

Figure 13 Tilt sensor outputs and assistive forces 
Through the innovative driving and control strategy, the developed robotic walker achieves walking assistance on a slope without the user's instructions as well as complex sensing and control. The robotic walker can effectively provide the user with an assistive force during the process of walking uphill and downhill via a simple mechanical structure and control method.

For safety purposes, additional functions of the robotic walker, such as braking and obstacle avoidance, will be investigated in future research.

\section{Conclusions}

A robotic walker with an APHA for slope mobility assistance was proposed to supply an assistive force when the user is walking on uphill and downhill roads.

The APHA consists mainly of an MR brake and a DC motor with a worm gear reducer. The MR brake used in the APHA was specifically designed based on the torque requirements of the robotic walker. A prototype of the APHA was constructed, and experiments were conducted to determine the relationships between the passive and active torques and the current. The experimental results show that controllable active and passive torque can be provided by the APHA, and that the torques from the APHA can satisfy the requirements of the robotic walker.

A robotic walker and its control algorithm were developed. A prototype of the robotic walker was constructed, and the human-robotic walker interaction force during walking on different road surfaces was measured. The experimental results show that no assistive force was supplied by the robotic walker when the user walked on an even road. In this case, the user moved the device using his own force, which implies that the robotic walker worked like a conventional walker. When walking on either an uphill or downhill slope, an appropriate assistive force can be provided by the robotic walker based on the gradient of the road through a simple control system and method. Slope mobility assistance is therefore successfully achieved.

\section{Acknowledgements}

Not applicable.

\section{Authors' Contributions}

$J \mathrm{~L}$ was in charge of the whole trial; $L Z$ wrote the manuscript; $T L$ assisted with sampling and laboratory analyses. All authors read and approved the final manuscript.

\section{Authors' Information}

Junqiang Li, born in 1979, is currently an associate professor at School of Mechanical Engineering, Hebei University of Technology, China. He received his bachelor degree and master degree in Mechatronics from Hebei University of Technology, China, in 2002 and 2005, respectively, and PhD in Mechatronics from Harbin Institute of Technology, China, in 2010. His research interests include man-machine system and intelligent robotics.
Lei Zhao, born in 1994, is currently a master candidate at School of Mechanical Engineering, Hebei University of Technology, China.

Tiejun Li, born in 1967, is currently a professor at School of Mechanical Engineering, Hebei University of Technology, China. He received his Master degree from Hebei University of Technology, China, in 1993, and PhD degree from Harbin Institute of Technology, China, in 1997. His research interests include manmachine system and intelligent robotics.

\section{Funding}

Supported by National Natural Science Foundation of China (Grant No. U1813222), and Hebei Provincial Natural Science Foundation of China (Grant No. E2018202316).

\section{Competing Interests}

The authors declare no competing financial interests.

Received: 29 May 2020 Revised: 5 February 2021 Accepted: 22 September 2021

Published online: 30 October 2021

\section{References}

[1] M Hirvensalo, T Rantanen, E Heikkinen, et al. Mobility difficulties and physical activity as predictors of mortality and loss of independence in the community-living older population. Journal of the American Geriatrics Society, 2000, 48(5): 493-498.

[2] T Gill, D Baker, M Gottschalk, et al. A prehabilitation program for the prevention of functional decline: effect on higher-level physical function. Archives of Physical Medicine and Rehabilitation, 2004, 85(7): 1043-1049.

[3] KWakita, J Huang, P Di, et al. Human-walking-intention-based motion control of an omnidirectional-type cane robot. IEEE/ASMETransactions on Mechatronics, 2013, 18(1): 285-296.

[4] HYWang, B Q Sun, XTWu, et al. An intelligent cane walker robot based on force control. The 5th Annual IEEE International Conference on Cyber Technology in Automation, Control and Intelligent Systems, Shenyang, China, June 8-12, 2015: 1333-1337.

[5] P Nazemzadeh, F Moro, D Fontanelli, et al. Indoor positioning of a robotic walking assistant for large public environments. IEEE Transactions on Instrumentation and Measurement, 2015, 64(11): 2965-2976.

[6] S Q Wang, LT Wang, C Meijneke, et al. Design and control of the MINDWALKER exoskeleton. IEEE Transactions on Neural Systems and Rehabilitation Engineering, 2015, 23(2): 277-286.

[7] D Ming, S L Jiang, Z P Wang, et al. Review of walk assistant exoskeleton technology: human-machine interaction. Acta Automatica Sinica, 2017, 43(7): 1089-1100. (in Chinese)

[8] N Zengin, $\mathrm{H}$ Zengin, B Fidan. Adaptive control of intelligent walker guided human-walker systems. IEEE International Symposium on Robotics and Intelligent Sensors, Ottawa, Canada, October 5-7, 2017: 186-191.

[9] C H Ko, KY Young, Y C Huang, et al. Active and passive control of walkassist robot for outdoor guidance. IEEE/ASME Transactions on Mechatronics, 2013, 18(3): 1211-1220.

[10] M Spenko, H Yu, S Dubowsky. Robotic personal aids for mobility and monitoring for the elderly. IEEE Transactions on Neural Systems and Rehabilitation Engineering, 2006, 14(3): 344-351.

[11] G Lee, T Ohnuma, N Y Chong, et al. Walking intent-based movement control for JAIST active robotic walker. IEEE Transactions on Systems, Man, and Cybernetics: Systems, 2014, 44(5): 665-672.

[12] J Paulo, P Peixoto, U J Nunes. ISR-AIWALKER: Robotic walker for intuitive and safe mobility assistance and gait analysis. IEEE Transactions on Human-Machine Systems, 2017, 47(6): 1110-1122.

[13] G Chalvatzaki, X S Papageorgiou, C S Tzafestas, et al. Augmented human state estimation using interacting multiple model particle filters with probabilistic data association. IEEE Robotics \& Automation Letters, 2018 : 1872-1879.

[14] C A Cifuentes, C Rodriguez, A Frizera-Neto, et al. Multimodal humanrobot interaction for walker-assisted gait. IEEE Systems Journal, 2016, 10(3): 933-943. 
[15] WXXu, J Huang, Q Y Yan, et al. Research on walking-aid robot motion control with both compliance and safety. Acta Automatica Sinica, 2016, 42(12): 1859-1873. (in Chinese)

[16] M Andreetto, S Divan, F Ferrari, et al. Combining Haptic and Bang-Bang braking actions for passive robotic walker path following. IEEE Transactions on Haptics, 2019, 12(4): 542-553.

[17] Y Hirata, A Hara, K Kosuge. Motion control of passive intelligent walker using servo brakes. IEEE Transactions on Robotics, 2007, 23(5): 981-990.

[18] T Kikuchi, T Tanaka, S Tanida, et al. Basic study on gait rehabilitation system with intelligently controllable walker (i-Walker). Proceedings of the IEEE International Conference on Robotics and Biomimetics, Tianjin, China, December 14-18, 2010: 277-282.

[19] N Nejatbakhsh, K Kosuge. User-environment based navigation algorithm for an omnidirectional passive walking aid system. Proceedings of the IEEE 9th International Conference on Rehabilitation Robotics, Chicago, IL, USA, June 28-July 1, 2005: 178-181.

[20] A G Leal, C R Diaz, M F Jimenez, et al. Polymer optical fiber based sensor system for smart walker instrumentation and health assessment. IEEE Sensors Journal, 2019, 19(2): 567-574.

[21] K M Lee, C H Lee, S W Hwang, et al. Power-Aassisted wheelchair with gravity and friction compensation. IEEE Transactions on Industrial Electronics, 2016, 63(4): 2203-2211.
[22] P Zhang, K H LEE, C H LEE. Friction behavior of magnetorheological fluids with different material types and magnetic field strength. Chinese Journal of Mechanical Engineering, 2016, 29(1): 84-90.

[23] I Fitrian, S A Mazlan, Z Hairi. A design and modelling review of rotary magnetorheological damper. Materials and Design, 2013, 51: 575-591.

[24] M Y Huang, E R Wang, F H Min. Chaotic vibration analysis of vehicle suspension system with magneto-rheological damper. Journal of Vibration and Shock, 2015, 34(24): 128-134. (in Chinese)

[25] C Rossa, A Jaegy, J Lozada, et al. Design considerations for magnetorheological brakes. IEEE/ASME Transactions on Mechatronics, 2014, 19(5): 1669-1680.

[26] W D Wang, T S Sun, X Q Yuan, et al. Design and analysis of variable flexible actuator for human-robot interaction. Journal of Northwestern Polytechnical University, 2019, 37(2): 39-45. (in Chinese)

[27] Q H Nguyen, S B Choi. Selection of magnetorheological brake types via optimal design considering maximum torque and constrained volume. Smart Materials \& Structures, 2012, 21(1): 015012.

[28] Y Gan, Z L Tian, H Dong et al. China materials engineering, Volume 2. Beijing: Chemical Industry Press, 2006: 93. (in Chinese)

\section{Submit your manuscript to a SpringerOpen ${ }^{\circ}$ journal and benefit from:}

- Convenient online submission

- Rigorous peer review

- Open access: articles freely available online

- High visibility within the field

- Retaining the copyright to your article

Submit your next manuscript at springeropen.com 